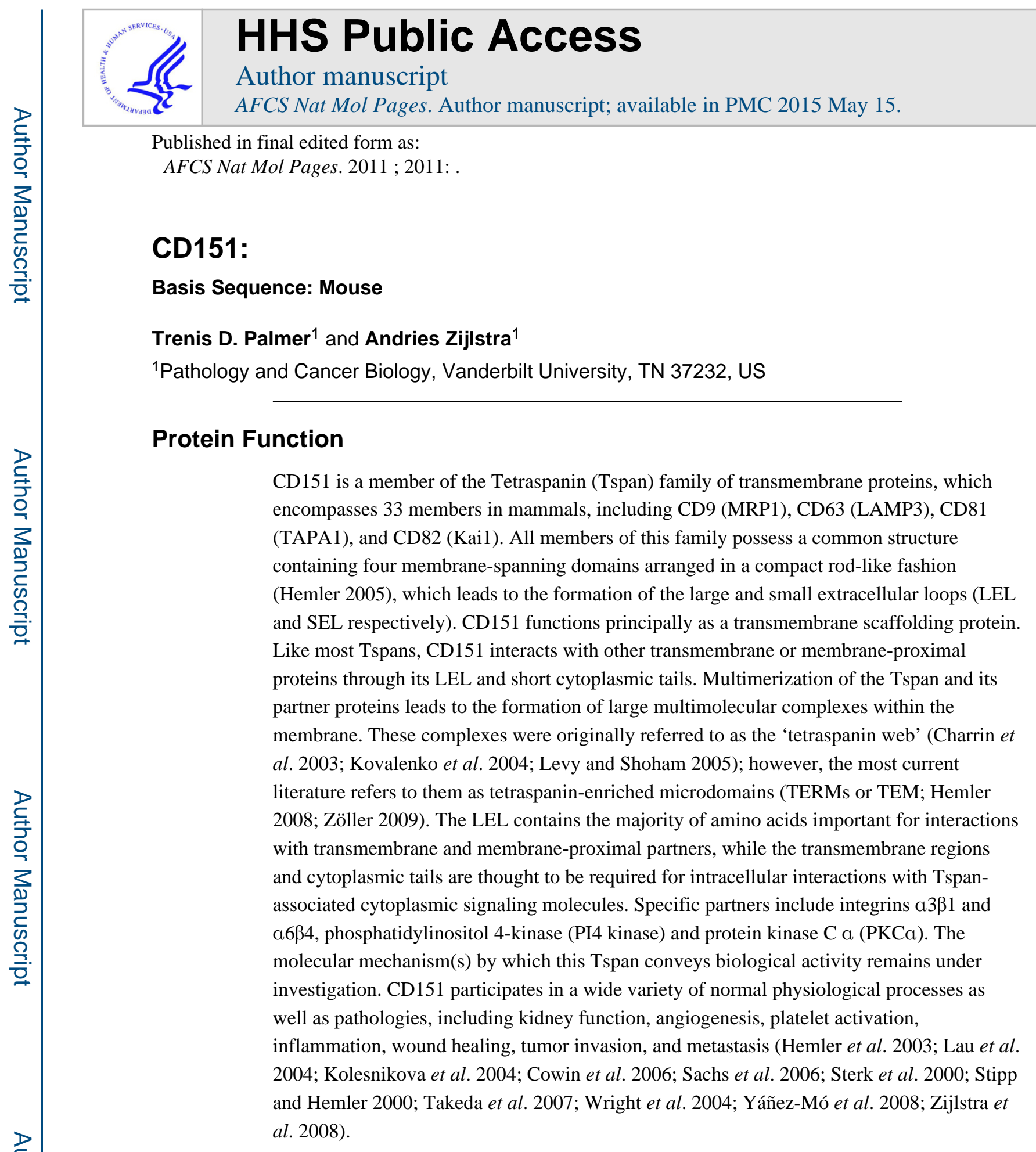

\title{
Regulation of Activity
}

Since Tspans are generally expected to function as scaffolding proteins, their activity is defined by their ability to integrate themselves and their partner proteins into macromolecular complexes. This activity of CD151 is primarily regulated posttranslationally through modifications of its subcellular distribution or the stability of its interaction with membrane partners. The most common post-translational modification 
involves the palmitoylation of conserved cysteine residues in the transmembrane domains by the palmitoyl-acyl transferase (PAT) DHHC2, one of 23 known mammalian PATs (Sharma et al. 2008). Palmitoylation of Tspan proteins such as CD151 affects the assembly and maintenance of TERMS, while also affecting Tspan subcellular distribution, stability during biosynthesis, cell signaling, motility, and morphology. It has also been shown that palmitoylation of CD151 is involved in the regulation of Tspan-Tspan interaction because palmitoylation-deficient CD151 is unable to complex with other Tspan family members (CD9, CD63, CD81) (Berditchevski et al. 2002; Kovalenko et al. 2004; Yang et al. 2002; Yang et al. 2004). The importance of this modification has been confirmed both by short interfering RNA (siRNA)-mediated knockdown of expression of DHHC2 as well as the expression of palmitoylation-deficient CD151 mutants. In addition to palmitoylation on conserved cysteine residues, CD151 extracellular domain is routinely glycosylated. While this post-translational modification is important for the modulatory ability of CD151 on a $3 \beta 1$ integrin, it is not required for the formation of the tetraspanin-integrin complex. Furthermore, it has been demonstrated that the loss of CD151 decreases migration on laminin 322, and this effect is reversed with the re-expression of wild-type CD151 but not a glycosylation-deficient CD151 mutant. These findings suggest that the glycosylation state of CD151 modulates integrin activity and function (Baldwin et al. 2008). Tspan clustering is the primary mechanism that can influence biological activity of these proteins. Clustering antibodies for CD151 and other Tspans have produced dramatic phenotypes including complete immobilization of tumor cells (Zijlstra et al. 2008). These experiments demonstrate that mechanisms capable of regulating Tspan clustering are potent regulators of Tspan activity.

\section{Interactions with Ligands and Other Proteins}

CD151 interacts with a large variety of proteins, including integrins, other tetraspanins, proteases, and signaling molecules. Their assembly into TERMs makes it somewhat difficult to evaluate isolated tetraspanin-partner interactions. The associations listed here are confirmed, direct interactions but it has been suggested that Tspans such as CD151 can interact with and regulate the function of many more proteins, directly or indirectly (Hemler 2005; Zöller 2009) and these associations are reviewed by Zijlstra in "Tetraspanins in Cancer", a chapter in Cell-Extracellular Matrix Interactions in Cancer (DOI: 10.1007/978-1-4419-0814-8_10 (2009)).

\section{Interactions with integrins}

The most well studied CD151 interactions are those that involve the laminin-binding integrins a $3 \beta 1$ and a $6 \beta 1$ (Kazarov et al. 2002), and these CD151-integrin complexes can regulate cell morphology and migration by controlling adhesion and adhesive strengthening (Sincock et al. 1999; Lammerding et al. 2003; Geary et al. 2008) and cytoplasmic signaling (Alvares et al. 2008; Chattopadhyay et al. 2003; Yamada et al. 2008a; Zhang et al. 2001). The nature, stoichiometry, and affinity of tetraspanin-partner interactions have been difficult to assess quantitatively. Direct partners of Tspans are identified through a combination of protein cross-linking, cell-surface biotinylation, differential detergent extraction, and reciprocal immunoprecipitation (Yauch et al. 1998; Charrin et al. 2003; Chattopadhyay et 
al. 2003; Yauch et al. 2000; Zhang et al. 2001). The CD151-a3 $\beta 1$ integrin complex is one of the best defined interactions. This complex can be immunoprecipitated under both mild and stringent detergent extraction conditions indicating a strong, primary interaction that is defined by the specific QRD binding sequence (Kazarov et al. 2002; Yamada et al. 2008b). The loss of CD151 in human mammary epithelial and carcinoma cells leads to migration deficiencies on laminin-rich matrixes (Novitskaya et al. 2010; Winterwood et al. 2006; Yang et al. 2010). In addition to manipulating the activity of its integrin partners directly, evidence has been presented that the tetraspanin can regulate a $3 \beta 1$ trafficking (Liu et al. 2007) and glycosylation (Baldwin et al. 2008). Together these observations provide strong support for both physical and functional roles for CD151 in a3 $\beta 1$ biology. The integrins a6 $\beta 1$ and a6 64 are also partners of CD151, although the interaction is not as stable as the CD151-a3 interaction (Sterk et al. 2000; Sterk et al. 2002). Disruption of CD151-a6 interaction does not alter integrin cell surface expression or cellular adhesion to laminin, but it does diminish a6-dependent cell spreading and integrin-mediated adhesive strengthening (Lammerding et al. 2003). The majority of biochemical and functional data have been generated with studies evaluating the ability of CD151 to regulate the functions of lamininbinding integrins $a 3$ and $a 6$. However, CD151 has also been demonstrated to interact with aIIb $\beta 3$ in both murine and human platelets under both mild and stringent detergent extraction conditions. The functional significance of this interaction was demonstrated using CD151 null platelets isolated from the CD151 knockout mouse. Characterization of the in vivo phenotype of these mice demonstrates a tendency to re-bleed and poor clot retraction. CD151 might therefore be required for potentiation of aIIb $\beta 3$ signaling events, but not the cell surface expression of the integrin as the latter is present at equivalent levels in both wild-type and CD151 knockout platelets (as demonstrated by flow cytometry) (Fitter et al. 1999; Lau et al. 2004). The integrin a $5 \beta 1$, another fibronectin receptor, can be coimmunoprecipitated from human platelets under mild detergent extraction conditions, whereas this complex is lost under stringent detergent extraction conditions. Although this complex is not as stable as the association with a $3 \beta 1$, the loss of CD151 decreases the adherence of human $\mathrm{T}$ cells to fibronectin, and anti-CD151 antibody inhibits $\mathrm{T}$ cell adherence to fibronectin but not collagen or laminin (Hasegawa et al. 1998). The molecular mechanism by which CD151 controls migration remains unknown. In vivo work with antiCD151 antibody indicates, however, that CD151 can have a broad impact on all matrixbinding integrins (Zijlstra et al. 2008).

\section{Interactions with signaling molecules}

A wealth of literature has suggested that the ability of CD151 to associate with intracellular signaling molecules is required for its ability to interact with and regulate various integrins. CD151 associates with many signaling molecules including PKCa, integrin $\beta \mathrm{II}$, and PI4 kinase type IIa (Shigeta et al. 2003; Yauch et al. 1998; Zhang et al. 2001), but the importance of these interactions is not fully known. It is reasonable to speculate, however, that the CD151 interactions with these signaling molecules could influence intracellular signaling by incorporating them into the TERM. It has been demonstrated that, in addition to interactions with the above mentioned signaling molecules, some of the proliferative effects associated with CD151 are due to its ability to activate extracellular signal-regulated kinases 1 and 2 (Erk1/2) and protein kinase B (Akt) signaling in non-tumorigenic HB2 mammary 
epithelial cells (Novitskaya et al. 2010). The loss of CD151 abrogates Erk1/2 and Akt signaling, and additionally rescues the ability of these cells to form luminal-like structures when grown in three-dimensional (3D) cultures, indicating that CD151 might be upstream of Erk1/2 activation. CD151 has been suggested to influence small GTPase activity, including Cdc42 and RhoA activation (Shigeta et al. 2003; Johnson et al. 2009). In CD151 null carcinoma cells the loss of CD151 leads to an increase in cell migration and a loss of cellcell adhesion that is independent of the loss of E-cadherin. Re-expression of CD151 promotes the reestablishment of normal cell-cell junctions. Interestingly, this activity is dependent on the CD151/a3 31 complex because in carcinoma cells expressing a CD151 mutant that cannot associate with a 3 the cell-cell junctions remain disorganized and Ecadherin is mislocalized (Johnson et al. 2009).

\section{Interactions with proteases}

Several matrix metalloproteinases have been associated with CD151, including MMP7 (Shiomi et al. 2005), MMP14 (Yáñez-Mó et al. 2008) and MMP9 (Hong et al. 2006).

Although the importance of the interactions of CD151 with matrix metalloproteinases is still poorly understood, these studies indicate that the tetraspanin may control activation and localization of these proteases.

The incorporation of CD151 into TERMs is likely to allow it to interact directly and indirectly with a large number of proteins and regulate their function. The extent to which this activity influences biological function will have to be determined for each individual biological system.

\section{Regulation of Concentration}

Mechanisms by which the expression of CD151 is regulated are not completely understood, but an interaction with the E3 ubiquitin ligase GRAIL (gene related to anergy in lymphocytes) was recently identified. This interaction targets CD151 for proteasomalmediated degradation (Lineberry et al. 2008). Recently it has been suggested that hypoxia is able to regulate CD151 expression and potentially alter CD151-mediated adhesion of tumor cells. The ability of hypoxia to downregulate CD151 expression is due to the activation of a hypoxia response element (HRE) located in the CD151 promoter region (Chien et al. 2008). Transcriptional regulation of CD151 is also achieved by Sp1, which is responsible for the basal activation of CD151 and chromatin accessibility of the CD151 promoter (Wang et al. 2010). Within individual cells the CD151 concentration is controlled by subcellular distribution. Within tissues CD151 is nearly ubiquitously expressed, but present at elevated levels in endothelial cells, platelets, and epithelial cells that contact basement membranes. CD151 expression is frequently altered in tumor cells, although the mechanism is unknown.

\section{Subcellular Localization}

In normal tissues and confluent endothelial or epithelial cell cultures CD151 is predominantly localized to the basolateral surface and to areas of cell-cell contact (Chattopadhyay et al. 2003; Huang et al. 2005; Shigeta et al. 2003; Sincock et al. 1999; Yáñez-Mó et al. 1998). In individually cultured cells without cell-cell contact or non- 
adherent cells, CD151 is frequently internalized into endosomal and lysosomal compartments (Liu et al. 2007; Sincock et al. 1999) as a component of the endocytic pathway. In contrast, tumor cells frequently exhibit general cell surface distribution and localization in cytoplasmic vesicles. In epithelial cells, CD151 is also seen in hemidesmosome-like structures, where it associates with a6ß34 (Sterk et al. 2000; Wright et al. 2004). More recently, CD151, along with other tetraspanins, has been shown to be enriched in exosomes (Hemler 2003; Zöller 2009).

\section{Major Sites of Expression}

Although CD151 was originally identified as a platelet cell surface antigen (Ashman et al. 1991; Fitter et al. 1995), it is expressed in nearly all tissues. It is abundantly expressed in many cells of the vascular system, including endothelial cells, megakaryocytes and platelets. In normal breast CD151 is expressed in myoepithelial cells, while high levels of expression are seen in ductal epithelial cells of the colon and prostate. CD151 is also highly expressed in the glomeruli and tubules of the kidney, the basal epithelia of the skin, cardiomyocytes, and Schwann cells of the peripheral nervous system (Sincock et al. 1997; Yang et al. 2008). CD151 is also expressed in most solid tumors and upregulated in many aggressive neoplasia. In contrast, it is distinctly absent from lymphoma.

Expression patterns for CD151 can be reviewed effectively at Proteinatlas.

\section{Phenotypes}

In humans, genetic mutations in CD151 result in hereditary nephrotic syndrome, which ultimately leads to end-stage renal failure. These patients also suffer from sensorineural deafness, skin blistering, and thalassemia, a blood disorder characterized by abnormal hemoglobin production (Wright et al. 2004; Karamatic Crew et al. 2004; Sachs et al. 2006). Several CD151 knockout mice have been generated to further investigate the biological function of this Tspan (Baleato et al. 2008b; Sachs et al. 2006; Wright et al. 2004). CD151 null mice on a B6 background were generated using the Cre recombinase system. Due to the known associations of CD151 with integrin family members, it was anticipated that the loss of CD151 would lead to a loss or alteration in cell surface integrin expression in various isolated cells and tissues, but integrin expression was unchanged in the absence of CD151. It was also anticipated that the formation of hemidesmosomes would be altered in CD151 null mice due to the strong associations of CD151 with a6 $\beta 4$, but the expression of the integrin and the formation of these structures was unchanged (Wright et al. 2004). Initial phenotypic characterization of the mice showed that they are healthy, viable, and fertile. Although the phenotype of the CD151 null mice is relatively mild, they do have a minor bleeding deficiency characterized by a decrease in bleeding time endpoint coupled with an increased tendency to re-bleed when compared with CD151 wild-type mice. Although these data suggest that the CD151 null mice are relatively healthy, in addition to minor defects in hemostasis they exhibit alterations in skin wound healing both in vitro and in vivo (Orlowski et al. 2009; Wright et al. 2004; Cowin et al. 2006). Interestingly, T cell activation is also diminished in CD151 null mice due to defects in dendritic cell co-stimulation (Sheng et al. 2009). In contrast with the B6 background, CD151 knockout mice on a mixed background 
(FVB/Nx129 and the FVB/B6) develop severe kidney disease with pronounced proteinuria and glomerulosclerosis (Sachs et al. 2006). These mice have served as suitable models to investigate the role of CD151 in the kidney and the pathologies associated with the development of glomerulosclerosis due to the loss of CD151. A strain-dependent penetrance phenotype has subsequently been described (Baleato et al. 2008a) and may be indicative of compensatory mechanisms in CD151-deficient mouse models.

\section{Splice Variants}

While no splice variants have been found in the human CD151 sequence, alternative splicing of murine CD151 generates three transcripts with different $5^{\prime}$ untranslated regions (Fitter et al. 1998).

\section{Antibodies}

A majority of the information currently known about CD151 has been generated using specific monoclonal antibodies generated against the protein. Although all of these antibodies bind CD151, they do not recognize it when it is contained in certain protein complexes (Serru et al. 1999; Geary et al. 2001; Yamada et al. 2008), and furthermore tissue staining patterns can differ between antibodies (Geary et al. 2001). Below is a list of the commonly used and referenced antibodies.

mAbs against human CD151 include:

1A5 (Testa et al. 1999; Zijlstra et al. 2008)

$8 C 3$ (Nishiuchi et al. 2005)

SFA1.2B4 (Charrin et al. 2001)

TS151 (Serru et al. 1999)

TS151r (Serru et al. 1999)

LIA1/1 (Yáñez-Mó et al. 1998)

VJ1/16 (Yáñez-Mó et al. 1998)

11B1 (Geary et al. 2001)

14B1 (Hasegawa et al. 1996)

5C11 (Yauch et al. 2000)

14A2.H1 (Ashman et al. 1991)

The epitope binding and staining patterns of many of these antibodies have been documented by the laboratories of Ashman and Sekiguchi (Yamada et al. 2008b; Geary et al. 2001).

$11 \mathrm{G} 5$ is the most commonly available commercial antibody. 


\section{References}

Alvares SM, Dunn CA, Brown TA, Wayner EE, Carter WG. The role of membrane microdomains in transmembrane signaling through the epithelial glycoprotein Gp140/CDCP1. Biochim Biophys Acta. Mar.2008 1780:3.

Ashman LK, Aylett GW, Mehrabani PA, Bendall LJ, Niutta S, Cambareri AC, Cole SR, Berndt MC. The murine monoclonal antibody, 14A2.H1, identifies a novel platelet surface antigen. Br J Haematol. Oct.1991 79:2.

Baldwin G, Novitskaya V, Sadej R, Pochec E, Litynska A, Hartmann C, Williams J, Ashman L, Eble JA, Berditchevski F. Tetraspanin CD151 regulates glycosylation of (alpha)3(beta)1 integrin. J Biol Chem. 2008; 283:51.

Baleato RM, Guthrie PL, Gubler MC, Ashman LK, Roselli S. Deletion of CD151 results in a straindependent glomerular disease due to severe alterations of the glomerular basement membrane. Am J Pathol. Oct.2008 173:4.

Berditchevski F, Odintsova E, Sawada S, Gilbert E. Expression of the palmitoylation-deficient CD151 weakens the association of alpha 3 beta 1 integrin with the tetraspanin-enriched microdomains and affects integrin-dependent signaling. J Biol Chem. 2002; 277:40. [PubMed: 11606568]

Charrin S, Le Naour F, Oualid M, Billard M, Faure G, Hanash SM, Boucheix C, Rubinstein E. The major CD9 and CD81 molecular partner. Identification and characterization of the complexes. $\mathrm{J}$ Biol Chem. 2001; 276:17.

Charrin S, Manié S, Billard M, Ashman L, Gerlier D, Boucheix C, Rubinstein E. Multiple levels of interactions within the tetraspanin web. Biochem Biophys Res Commun. 2003; 304:1. [PubMed: 12705874]

Chattopadhyay N, Wang Z, Ashman LK, Brady-Kalnay SM, Kreidberg JA. alpha3beta1 integrinCD151, a component of the cadherin-catenin complex, regulates PTPmu expression and cell-cell adhesion. J Cell Biol. 2003; 163:6.

Chien CW, Lin SC, Lai YY, Lin BW, Lin SC, Lee JC, Tsai SJ. Regulation of CD151 by hypoxia controls cell adhesion and metastasis in colorectal cancer. Clin Cancer Res. 2008; 14:24.

Cowin AJ, Adams D, Geary SM, Wright MD, Jones JC, Ashman LK. Wound healing is defective in mice lacking tetraspanin CD151. J Invest Dermatol. Mar.2006 126:3.

Fitter S, Seldin MF, Ashman LK. Characterisation of the mouse homologue of CD151 (PETA-3/ SFA-1); genomic structure, chromosomal localisation and identification of 2 novel splice forms. Biochim Biophys Acta. 1998; 1398:1. [PubMed: 9602019]

Fitter S, Sincock PM, Jolliffe CN, Ashman LK. Transmembrane 4 superfamily protein CD151 (PETA-3) associates with beta 1 and alpha IIb beta 3 integrins in haemopoietic cell lines and modulates cell-cell adhesion. Biochem J. Feb 15.1999 338(Pt 1)

Fitter S, Tetaz TJ, Berndt MC, Ashman LK. Molecular cloning of cDNA encoding a novel plateletendothelial cell tetra-span antigen, PETA-3. Blood. 1995; 86:4.

Geary SM, Cambareri AC, Sincock PM, Fitter S, Ashman LK. Differential tissue expression of epitopes of the tetraspanin CD151 recognised by monoclonal antibodies. Tissue Antigens. Sep. $200158: 3$.

Geary SM, Cowin AJ, Copeland B, Baleato RM, Miyazaki K, Ashman LK. The role of the tetraspanin CD151 in primary keratinocyte and fibroblast functions: implications for wound healing. Exp Cell Res. 2008; 314:11-12. [PubMed: 17678891]

Hasegawa H, Nomura T, Kishimoto K, Yanagisawa K, Fujita S. SFA-1/PETA-3 (CD151), a member of the transmembrane 4 superfamily, associates preferentially with alpha 5 beta 1 integrin and regulates adhesion of human $\mathrm{T}$ cell leukemia virus type 1-infected $\mathrm{T}$ cells to fibronectin. $\mathrm{J}$ Immunol. 1998; 161:6.

Hasegawa H, Utsunomiya Y, Kishimoto K, Yanagisawa K, Fujita S. SFA-1, a novel cellular gene induced by human T-cell leukemia virus type 1 , is a member of the transmembrane 4 superfamily. J Virol. May.1996 70:5.

Hemler ME. Tetraspanin functions and associated microdomains. Nat Rev Mol Cell Biol. Oct.2005 $6: 10$. 
Hemler ME. Targeting of tetraspanin proteins--potential benefits and strategies. Nat Rev Drug Discov. Sep.2008 7:9.

Hemler ME. Tetraspanin proteins mediate cellular penetration, invasion, and fusion events and define a novel type of membrane microdomain. Annu Rev Cell Dev Biol. 2003; 19

Hong IK, Jin YJ, Byun HJ, Jeoung DI, Kim YM, Lee H. Homophilic interactions of Tetraspanin CD151 up-regulate motility and matrix metalloproteinase-9 expression of human melanoma cells through adhesion-dependent c-Jun activation signaling pathways. J Biol Chem. 2006; 281:34.

Huang S, Yuan S, Dong M, Su J, Yu C, Shen Y, Xie X, Yu Y, Yu X, Chen S, Zhang S, Pontarotti P, $\mathrm{Xu} \mathrm{A}$. The phylogenetic analysis of tetraspanins projects the evolution of cell-cell interactions from unicellular to multicellular organisms. Genomics. Dec.2005 86:6.

Johnson JL, Winterwood N, DeMali KA, Stipp CS. Tetraspanin CD151 regulates RhoA activation and the dynamic stability of carcinoma cell-cell contacts. J Cell Sci. Jul 1.2009 122(Pt 13)

Karamatic Crew V, Burton N, Kagan A, Green CA, Levene C, Flinter F, Brady RL, Daniels G, Anstee DJ. CD151, the first member of the tetraspanin (TM4) superfamily detected on erythrocytes, is essential for the correct assembly of human basement membranes in kidney and skin. Blood. 2004; 104:8.

Kazarov AR, Yang X, Stipp CS, Sehgal B, Hemler ME. An extracellular site on tetraspanin CD151 determines alpha 3 and alpha 6 integrin-dependent cellular morphology. J Cell Biol. 2002; 158:7.

Kolesnikova TV, Stipp CS, Rao RM, Lane WS, Luscinskas FW, Hemler ME. EWI-2 modulates lymphocyte integrin alpha4beta1 functions. Blood. 2004; 103:8.

Kovalenko OV, Yang X, Kolesnikova TV, Hemler ME. Evidence for specific tetraspanin homodimers: inhibition of palmitoylation makes cysteine residues available for cross-linking. Biochem J. Jan 15.2004 377(Pt 2)

Lammerding J, Kazarov AR, Huang H, Lee RT, Hemler ME. Tetraspanin CD151 regulates alpha6beta1 integrin adhesion strengthening. Proc Natl Acad Sci U S A. 2003; 100:13.

Lau LM, Wee JL, Wright MD, Moseley GW, Hogarth PM, Ashman LK, Jackson DE. The tetraspanin superfamily member CD151 regulates outside-in integrin alphaIIbbeta3 signaling and platelet function. Blood. 2004; 104:8.

Levy S, Shoham T. Protein-protein interactions in the tetraspanin web. Physiology (Bethesda). Aug. 200520

Lineberry N, Su L, Soares L, Fathman CG. The single subunit transmembrane E3 ligase gene related to anergy in lymphocytes (GRAIL) captures and then ubiquitinates transmembrane proteins across the cell membrane. J Biol Chem. 2008; 283:42.

Liu L, He B, Liu WM, Zhou D, Cox JV, Zhang XA. Tetraspanin CD151 promotes cell migration by regulating integrin trafficking. J Biol Chem. 2007; 282:43.

Nishiuchi R, Sanzen N, Nada S, Sumida Y, Wada Y, Okada M, Takagi J, Hasegawa H, Sekiguchi K. Potentiation of the ligand-binding activity of integrin alpha3beta1 via association with tetraspanin CD151. Proc Natl Acad Sci U S A. 2005; 102:6.

Novitskaya V, Romanska H, Dawoud M, Jones JL, Berditchevski F. Tetraspanin CD151 regulates growth of mammary epithelial cells in three-dimensional extracellular matrix: implication for mammary ductal carcinoma in situ. Cancer Res. 2010; 70:11.

Orlowski E, Chand R, Yip J, Wong C, Goschnick MW, Wright MD, Ashman LK, Jackson DE. A platelet tetraspanin superfamily member, CD151, is required for regulation of thrombus growth and stability in vivo. J Thromb Haemost. Dec.2009 7:12.

Sachs N, Kreft M, van den Bergh Weerman MA, Beynon AJ, Peters TA, Weening JJ, Sonnenberg A. Kidney failure in mice lacking the tetraspanin CD151. J Cell Biol. 2006; 175:1.

Serru V, Le Naour F, Billard M, Azorsa DO, Lanza F, Boucheix C, Rubinstein E. Selective tetraspanintegrin complexes (CD81/alpha4beta1, CD151/alpha3beta1, CD151/alpha6beta1) under conditions disrupting tetraspan interactions. Biochem J. May 15.1999 340(Pt 1)

Sharma C, Yang XH, Hemler ME. DHHC2 affects palmitoylation, stability, and functions of tetraspanins CD9 and CD151. Mol Biol Cell. Aug.2008 19:8. [PubMed: 17942602]

Sheng KC, van Spriel AB, Gartlan KH, Sofi M, Apostolopoulos V, Ashman L, Wright MD. Tetraspanins CD37 and CD151 differentially regulate Ag presentation and T-cell co-stimulation by DC. Eur J Immunol. Jan.2009 39:1. 
Shigeta M, Sanzen N, Ozawa M, Gu J, Hasegawa H, Sekiguchi K. CD151 regulates epithelial cell-cell adhesion through PKC- and Cdc42- dependent actin cytoskeletal reorganization. J Cell Biol. 2003; $163: 1$.

Shiomi T, Inoki I, Kataoka F, Ohtsuka T, Hashimoto G, Nemori R, Okada Y. Pericellular activation of proMMP-7 (promatrilysin-1) through interaction with CD151. Lab Invest. Dec.2005 85:12.

Sincock PM, Fitter S, Parton RG, Berndt MC, Gamble JR, Ashman LK. PETA-3/CD151, a member of the transmembrane 4 superfamily, is localised to the plasma membrane and endocytic system of endothelial cells, associates with multiple integrins and modulates cell function. J Cell Sci. Mar. 1999 112(Pt 6)

Sincock PM, Mayrhofer G, Ashman LK. Localization of the transmembrane 4 superfamily (TM4SF) member PETA-3 (CD151) in normal human tissues: comparison with CD9, CD63, and alpha5beta1 integrin. J Histochem Cytochem. Apr.1997 45:4.

Sterk LM, Geuijen CA, Oomen LC, Calafat J, Janssen H, Sonnenberg A. The tetraspan molecule CD151, a novel constituent of hemidesmosomes, associates with the integrin alpha6beta4 and may regulate the spatial organization of hemidesmosomes. J Cell Biol. 2000; 149:4.

Sterk LM, Geuijen CA, van den Berg JG, Claessen N, Weening JJ, Sonnenberg A. Association of the tetraspanin CD151 with the laminin-binding integrins alpha3beta1, alpha6beta1, alpha6beta4 and alpha7beta1 in cells in culture and in vivo. J Cell Sci. Mar 15.2002 115(Pt 6)

Stipp CS, Hemler ME. Transmembrane-4-superfamily proteins CD151 and CD81 associate with alpha 3 beta 1 integrin, and selectively contribute to alpha 3 beta 1 -dependent neurite outgrowth. J Cell Sci. Jun.2000 113(Pt 11)

Takeda Y, Kazarov AR, Butterfield CE, Hopkins BD, Benjamin LE, Kaipainen A, Hemler ME. Deletion of tetraspanin Cd151 results in decreased pathologic angiogenesis in vivo and in vitro. Blood. 2007; 109:4.

Testa JE, Brooks PC, Lin JM, Quigley JP. Eukaryotic expression cloning with an antimetastatic monoclonal antibody identifies a tetraspanin (PETA-3/CD151) as an effector of human tumor cell migration and metastasis. Cancer Res. 1999; 59:15.

Wang J, Liu X, Ni P, Gu Z, Fan Q. SP1 is required for basal activation and chromatin accessibility of CD151 promoter in liver cancer cells. Biochem Biophys Res Commun. 2010; 393:2.

Winterwood NE, Varzavand A, Meland MN, Ashman LK, Stipp CS. A critical role for tetraspanin CD151 in alpha3beta1 and alpha6beta4 integrin-dependent tumor cell functions on laminin-5. Mol Biol Cell. Jun.2006 17:6.

Wright MD, Geary SM, Fitter S, Moseley GW, Lau LM, Sheng KC, Apostolopoulos V, Stanley EG, Jackson DE, Ashman LK. Characterization of mice lacking the tetraspanin superfamily member CD151. Mol Cell Biol. Jul.2004 24:13.

Yamada M, Sumida Y, Fujibayashi A, Fukaguchi K, Sanzen N, Nishiuchi R, Sekiguchi K. The tetraspanin CD151 regulates cell morphology and intracellular signaling on laminin-511. FEBS J. Jul.2008 275:13.

Yamada M, Tamura Y, Sanzen N, Sato-Nishiuchi R, Hasegawa H, Ashman LK, Rubinstein E, YáñezMó M, Sánchez-Madrid F, Sekiguchi K. Probing the interaction of tetraspanin CD151 with integrin alpha 3 beta 1 using a panel of monoclonal antibodies with distinct reactivities toward the CD151-integrin alpha 3 beta 1 complex. Biochem J. 2008; 415:3.

Yáñez-Mó M, Alfranca A, Cabañas C, Marazuela M, Tejedor R, Ursa MA, Ashman LK, de Landázuri MO, Sánchez-Madrid F. Regulation of endothelial cell motility by complexes of tetraspan molecules CD81/TAPA-1 and CD151/PETA-3 with alpha3 beta1 integrin localized at endothelial lateral junctions. J Cell Biol. 1998; 141:3.

Yañez-Mó M, Barreiro O, Gonzalo P, Batista A, Megías D, Genís L, Sachs N, Sala-Valdés M, Alonso MA, Montoya MC, Sonnenberg A, Arroyo AG, Sánchez-Madrid F. MT1-MMP collagenolytic activity is regulated through association with tetraspanin CD151 in primary endothelial cells. Blood. 2008; 112:8.

Yang X, Claas C, Kraeft SK, Chen LB, Wang Z, Kreidberg JA, Hemler ME. Palmitoylation of tetraspanin proteins: modulation of CD151 lateral interactions, subcellular distribution, and integrin-dependent cell morphology. Mol Biol Cell. Mar.2002 13:3. 
Yang X, Kovalenko OV, Tang W, Claas C, Stipp CS, Hemler ME. Palmitoylation supports assembly and function of integrin-tetraspanin complexes. J Cell Biol. 2004; 167:6.

Yang XH, Flores LM, Li Q, Zhou P, Xu F, Krop IE, Hemler ME. Disruption of laminin-integrinCD151-focal adhesion kinase axis sensitizes breast cancer cells to ErbB2 antagonists. Cancer Res. 2010; 70:6.

Yang XH, Richardson AL, Torres-Arzayus MI, Zhou P, Sharma C, Kazarov AR, Andzelm MM, Strominger JL, Brown M, Hemler ME. CD151 accelerates breast cancer by regulating alpha 6 integrin function, signaling, and molecular organization. Cancer Res. 2008; 68:9. [PubMed: 18172289]

Yauch RL, Berditchevski F, Harler MB, Reichner J, Hemler ME. Highly stoichiometric, stable, and specific association of integrin alpha3beta1 with CD151 provides a major link to phosphatidylinositol 4-kinase, and may regulate cell migration. Mol Biol Cell. Oct.1998 9:10.

Yauch RL, Hemler ME. Specific interactions among transmembrane 4 superfamily (TM4SF) proteins and phosphoinositide 4-kinase. Biochem J. Nov 1.2000 351(Pt 3)

Yauch RL, Kazarov AR, Desai B, Lee RT, Hemler ME. Direct extracellular contact between integrin alpha(3)beta(1) and TM4SF protein CD151. J Biol Chem. 2000; 275:13.

Zhang XA, Bontrager AL, Hemler ME. Transmembrane-4 superfamily proteins associate with activated protein kinase $\mathrm{C}$ (PKC) and link PKC to specific beta(1) integrins. J Biol Chem. 2001; 276:27.

Zijlstra A, Lewis J, Degryse B, Stuhlmann H, Quigley JP. The inhibition of tumor cell intravasation and subsequent metastasis via regulation of in vivo tumor cell motility by the tetraspanin CD151. Cancer Cell. Mar.2008 13:3. [PubMed: 18167333]

Zöller M. Tetraspanins: push and pull in suppressing and promoting metastasis. Nat Rev Cancer. Jan. 2009 9:1. 